\title{
Heralded Single Photons from a Silicon CROW Device (Project Report 1160881)
}

\author{
SHAYAN MOOKHERJEA \\ Department of Electrical and Computer Engineering, University of California, San Diego, MC 0407 La \\ Jolla CA 92093-0407 USA \\ Email:smookher@ucsd.edu
}

\section{Introduction and Goals}

This NSF-funded project [1160881] is a one-year supplement to NSF-funded project 0925133. The device structure that was studied is the CROW: Coupled Resonator Optical Waveguide, which was designed and fabricated using coupled microring resonators in silicon photonics. In this project, the CROW device was used to study entangled photon-pair generation and heralded singlephoton generation using spontaneous four-wave mixing (SFWM) which is based on the optical Kerr effect [1]. We used the CROW device structure as the foundation of this project because it offers a high four-wave mixing nonlinearity based on the triple resonance of the pump, signal and idler modes. For a typical silicon single-mode waveguide at $1550 \mathrm{~nm}$, the four-wave mixing nonlinear coefficient is $\gamma=200 \mathrm{~W}^{-1} \mathrm{~m}^{-1}$. A 35-ring CROW was measured to have a slow-light enhanced value $\gamma_{\mathrm{eff}}=4150 \mathrm{~W}^{-1} \mathrm{~m}^{-1}$ and there can be multiple passbands within the telecommunications window with such a high nonlinearity [2]. Since the pair-generation rate (PGR) in SFWM is quadratically proportional to $\gamma$, the improvement in $\gamma$ by a factor of 50 compared to a conventional waveguide can potentially lead to a $34 \mathrm{~dB}$ improvement in the PGR.

However, loss and deviations from phase matching reduce the PGR. Although tight-binding models of band-center propagation exist[3], propagation in the regions of high dispersion and high nonlinearity are sensitive to disorder [4], [5]. Measurements made in this project were compared to earlier theoretical models [6]-[8] and newer models developed here. Beyond the applications of this project, the CROW structure can have other applications including lowpower nonlinear optics [9]-[11] and lasers with novel properties [12]. There are also other scientific reasons to study the optical Kerr nonlinearity which is used as the physical principle for four-wave mixing, since it is also important in other areas of nonlinear optics such as the study of spatial and temporal solitons in both coupled and conventional waveguides [13]-[16].

\section{Activities}

As shown in Figure 1, the CROW structures which we used are based on microring (racetrack) resonators. These devices were fabricated by IBM 
(Yorktown Heights) under a collaborative project (1028553) supported by the NSF. This proposal requests funding to allow a graduate student researcher, Mr. Junrong Ong, and the PI, Associate Prof. Shayan Mookherjea, to visit and experimentally work alongside Dr. Kartik Srinivasan and post-doctoral researcher, Dr. Marcelo Davanco, at NIST to measure the photon pair generation properties, via spontaneous four-wave mixing experiments.

In these structures, the coupling between adjacent resonators is implemented using directional couplers. Previously, we have experimentally studied the dispersion of such couplers using a microring coupled to a waveguide [17], which is important to know. An accurate knowledge of dispersion is also necessary when CROWs are used as filters [18] or amplifiers [19]. Towards this end, we developed an extension of coupled-mode theory which is applicable to directional couplers in silicon photonics, coupled-waveguide structures [20] as well as coupled-resonator structures [21]. To increase pairgeneration rates, the SFWM properties will be measured in CROWs at relatively high pump power levels. In the high pump power regime, silicon microring resonators with directional couplers can be affected by some phenomena that are not always desirable for CROWs, such as bistability [22].

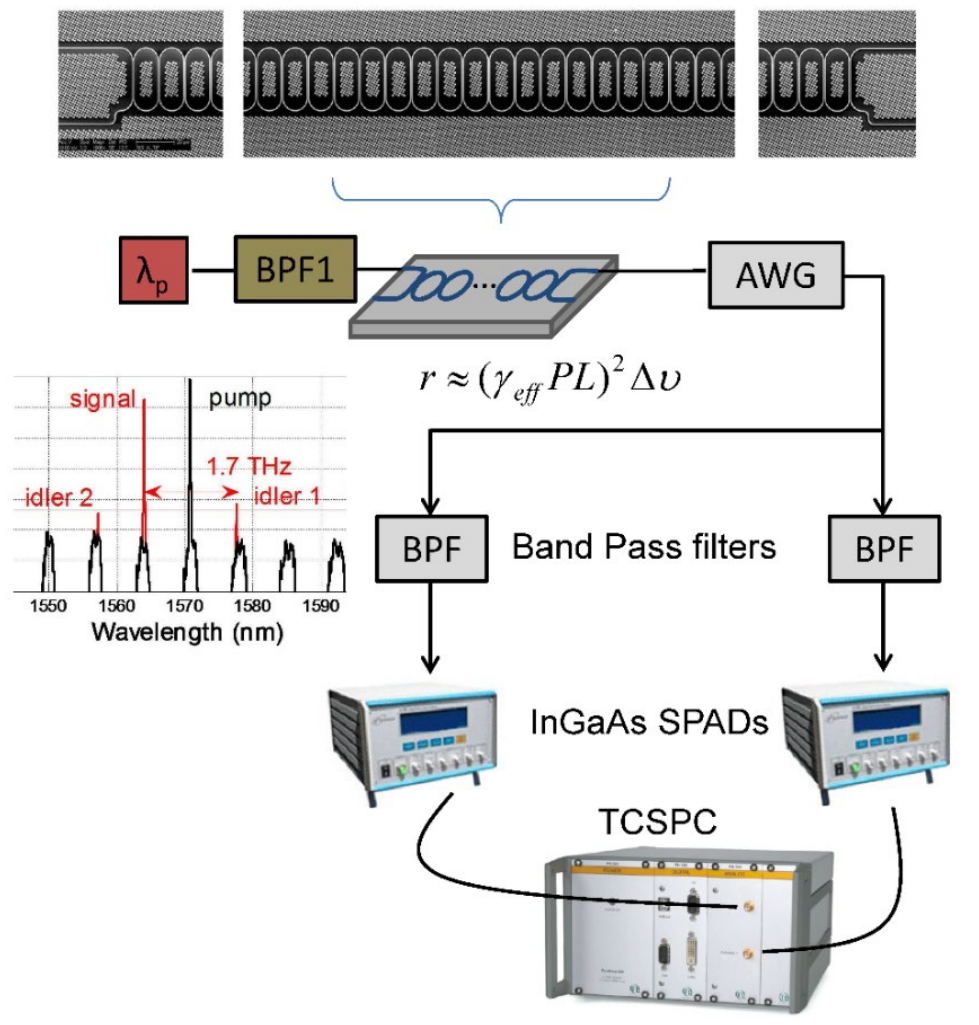

Figure 1 Experimental configuration for generating photon pairs through spontaneous four-wave mixing. The inset shows an experimental measurement of conventional four-wave mixing, showing the generation of two idlers. 


\section{Progress and Impact}

The milestones for this collaborative work with NIST in three-month increments were:

a) Develop a model for SFWM-based photon-pair generation in CROWs, leading to improved predictions of coincidence-to-accidental ratio (CAR).

b) Perform SFWM measurements and characterize the pair generation rate (PGR). Compare PGR in CROWs and conventional silicon waveguides.

c) Heralded single-photon generation: perform $\mathrm{g}^{(2)}$ measurements.

d) Dissemination and presentation of results: Publication of journal papers and conference presentations.

The experimental apparatus used for measuring photon pairs and heralded single photons are shown in Figures 1 and 2, respectively. Light from an tunable-wavelength external cavity semiconductor diode laser was used as the pump.

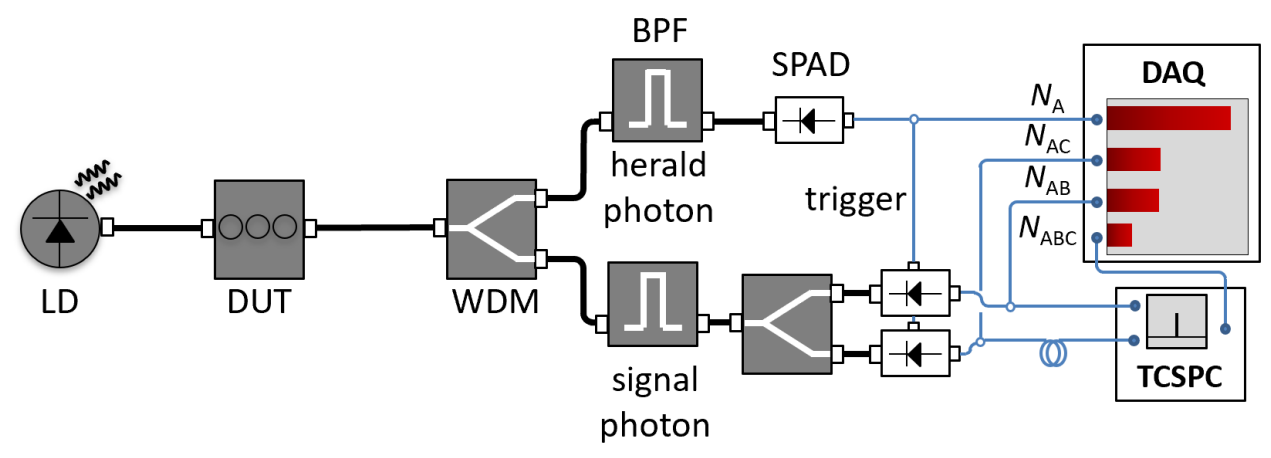

Figure 2 Configuration for studying heralded single-photon generation

A model for SFWM was developed using the Collett-Gardiner approach, and published in an Optics Express paper [23]. This method formulates the equations of motion in the time domain, in the Heisenberg picture. We solved the equations in the case of a single resonator as well as for multiple resonators, showing the potential advantages of the latter. We also indicated that the CROW structure could be used to potentially generate higher-dimensional entanglement, with a Schmidt number substantially greater than 1 .

In SFWM, two photons from the pump field are jointly annihilated to generate photon pairs consisting of one photon each in the Stokes and anti-Stokes modes. This is shown in Figure 3 (left panel). The transmission spectrum of the CROW is shown using a gray line. The black, red and blue lines show the transmission spectrum of a CW laser tuned to the wavelengths used by the pump, idler and signal modes, respectively. Energy conservation requires that $2 \omega_{\mathrm{P}}=\omega_{\mathrm{S}}+\omega_{\mathrm{I}}$. Phase matching is also satisfied since the three modes are in the same relative 
position within the CROW passband, and the Bloch momentum vectors are automatically matched. In a CROW, the Bloch dispersion relationship formed by the nearest-neighbor coupling of microring resonators dominates the dispersion relationship of the constituent waveguide [24].
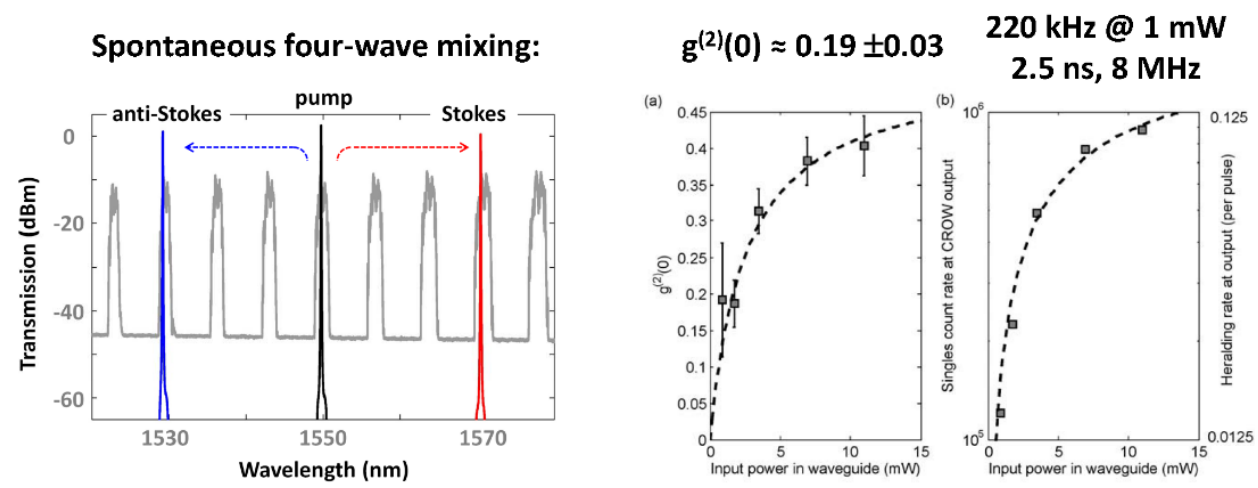

Figure 3 Spontaneous four-wave mixing in a CMOS-compatible silicon photonic device leads to the first report of heralded single photons from a silicon device.

The two-photon state that is generated is a superposition of a vacuum state and a two-photon state, and can be described as

$$
|\psi\rangle=|0\rangle_{s}|0\rangle_{a}+g \iint d \omega_{s} d \omega_{a} \eta\left(\omega_{s}, \omega_{a}\right)\left|\omega_{s}\right\rangle_{s}\left|\omega_{i}\right\rangle_{a}
$$

where the subscripts $s$ and $a$ refer to the Stokes and anti-Stokes frequencies, (also known as the signal and idler frequencies) and $g$ is the parametric gain coefficient, which is proportional to the PGR. The function $\eta$ describes the joint-spectral amplitude of the two-photon state, and the joint-spectral intensity, $|\eta|^{2}$ can be measured experimentally. For narrow-spectral-width pump waves in a single-polarization, single-spatial mode structure such as a CROW, frequency is the only continuous-variable degree of freedom for entanglement. A source can be designed for a large degree of entanglement and high degree of temporal synchronization between the Stokes and anti-Stokes photons.

We reported the first demonstration of heralded single photon generation in a silicon-based photonic device [25]. The experiment used a CROW waveguide which achieved good performance in four-wave mixing. A FWM experiment was also performed to show that the conversion efficiency of a 35-ring CROW was $20 \mathrm{~dB}$ higher than a straight waveguide of equal length. Next, we generated photon pairs through SFWM, as had recently been shown by a few groups. However, photon pair generation by itself is not sufficient for demonstrating single photon generation. One must implement heralding, a process by which 
one photon of the pair is detected and projects the system into a single photon Fock state, whose character must then be confirmed through a photon correlation measurement. We performed these measurements and demonstrated photon antibunching of the heralded signal $\left[\mathrm{g}^{(2)}(0)=0.19\right]$, an unambiguous signature of single photon generation. Lower values of $g^{(2)}(0)$ were achieved in later experiments after improvements in both the device quality and measurement apparatus.

The pair generation rate (PGR), or number of photon pairs generated per second, in a CROW depends upon the device geometry parameters, such as the axial periodicity $\Lambda=13.8 \mu \mathrm{m}$, effective racetrack radius $\mathrm{R}_{\mathrm{eff}}=\mathrm{R}+\mathrm{L}_{\mathrm{c}} / \pi$ (where $\mathrm{R}=6.5 \mu \mathrm{m}$ is the arc bending radius and $\mathrm{L}_{\mathrm{c}}=20 \mu \mathrm{m}$ is the length of the directional waveguide coupler between adjacent microrings) and a geometric parameter $\Gamma=\pi \mathrm{R}_{\mathrm{eff}} / \Lambda=2.9$. In the low pump power regime, the PGR is

$P G R=\frac{s_{s}+\Gamma}{s_{i}+\Gamma}\left(S_{i} \frac{s_{p} / \Gamma+1}{2}\right)^{2}\left(\gamma_{0} \mathrm{P} \overline{\mathrm{L}}\right)^{2} e^{-\alpha \mathrm{L}} \Delta \mathrm{f}$,

where S's are the slowing factors (ratio of the group index of the CROW to that of a conventional nanowire waveguide) at the pump (p), signal (s) and idler (i) wavelengths. The effective propagation length $\bar{L}=\left(1-e^{-\alpha L}\right) / \alpha$ is related to the geometric length $\mathrm{L}=\mathrm{N} \Lambda$ renormalized by the loss coefficient, $\alpha$.

For each passband, its band-center slowing factor $\mathrm{S}$ is inversely proportional to the magnitude of the inter-resonator coupling coefficient $|\kappa|, S=\Gamma /|\kappa|$. The coupling coefficient can be estimated from the passband width and its value varies with wavelength. For the device used in this experiment, values ranged from $|\kappa|^{2}=0.33$ near $1530 \mathrm{~nm}$ to $|\kappa|^{2}=0.49$ near $1600 \mathrm{~nm}$. The corresponding values of band-center $S$ varied from 5.14 at $1523 \mathrm{~nm}$ to 4.1 at $1607 \mathrm{~nm}$. The band-edge values of S are typically a factor of 2-3 higher, and for the specific pump, Stokes (signal) and anti-Stokes (idler) wavelengths that were used in these measurements, we estimate $\mathrm{S}_{\mathrm{p}}=12.1, \mathrm{~S}_{\mathrm{s}}=12.3$ and $\mathrm{S}_{\mathrm{i}}=11.1$. Thus, the effective four-wave mixing nonlinearity is

$\gamma_{\mathrm{eff}}=\gamma_{0} \frac{s_{i} s_{i}+s_{i} \Gamma}{2 \Gamma} \sqrt{\frac{s_{s}+\Gamma}{s_{i}+\Gamma}}=4150 \mathrm{~W}^{-1} \mathrm{~m}^{-1}$.

Compared to photonic crystals, which are also slow-wave waveguides [26], [27], (i) A single CROW waveguide can readily achieve multiple bands of slow and filtered light in the same physical structure, (ii) CROWs have among the highest measured nonlinearities of any type of slow-light waveguide, benefitting from the longitudinal confinement of the field, in addition to the usual transverse field confinement in conventional and photonic crystal waveguides, (iii) Certain types of CROWs may be advantageous from the point of view of integrating with other on-chip or off-chip lightwave circuitry. In particular, microring-based CROWs use the same waveguide cross-section as 
in conventional single-mode nanowire waveguides, which have reached a high level of maturity.

A collaboration with IBM's silicon photonics group provided insights into silicon photonics fabrication and also supported technical collaboration on fabricating the CROW structures [7], [28], [29]. Dr. Ivan B. Divliansky (Research Scientist, CREOL, Florida) collaborated with us for the electronbeam lithography of long waveguide structures [30], [31]. CROWs of up to 235 coupled resonators were successfully fabricated and measured, and a good agreement was achieved between experiments and theory [32]-[34].

Experimental details: Tapered and lensed fibers were used in conjunction with SU8 polymer spot-size converters for efficient on-chip coupling. By comparing midband-averaged transmission through waveguides and CROWs of different lengths, we measured the coupling losses to be $-4 \mathrm{~dB} /$ facet and the straight waveguide and CROW losses to be $-2.6 \mathrm{~dB} / \mathrm{cm}$ and $-0.21 \mathrm{~dB} /$ ring respectively. Infrared imaging is a useful experimental tool to study light propagation through the length of these structures [30], [35]. The filtering and separation of the signal and idler photons indicated in Fig. 1 and Fig. 3 was performed using fiber-pigtailed DWDM (dense wavelength division multiplexing) components. Once the signal and idler wavelengths were determined from a preliminary measurement of classical FWM, a set of DWDM filters was procured from OEQuest and spliced together. Each set of filters consisted of three cascaded DWDM filters, and was measured to have about $2.7 \mathrm{~dB}$ insertion loss, $1 \mathrm{~nm}$ bandwidth and should have at least $150 \mathrm{~dB}$ out-of-band rejection. A New Focus laser was used as the pump, amplified by a two-stage EDFA (Pritel). To reduce the ASE noise, the amplified laser was filtered by a 3-stage DWDM cascade, similar to those used after the chip, and a Yenista XTM50 filter instrument. An optical attenuator (JDS HA9) was used to control the power level coupled to the chip (typical value: $10-20 \mathrm{dBm}$ ).

Impact on Human Resources: One graduate student in the PI's group worked on this topic as part of their education and training. Part of this research was conducted at NIST (Gaithersburg, MD) in the laboratory of Dr. Kartik Srinivasan with the assistance of Dr. Marcelo Davanco. We are grateful to Andrea Bahgat Shehata and Alberto Tosi for making SPAD detectors available for the project. We are also grateful to Yenista for loaning a tunable filter instrument (XTM50) and to Dr. Vikas Anant for collaboration on single-photon detection apparatus, leading to a jointly-authored publication [36].

\section{Subsequent Extensions}

Novel techniques for measuring the transmission of light through CROWs with high spectral resolution were developed based on a single-scan, Jones matrixbased, interferometric measurement instrument (Luna Tech. Optical Vector 
Analyzer OVA5000, generously loaned by Luna Technologies, VA. A jointlyauthored publication resulted from this collaboration [7].

Microring resonators are sensitive to disorder but trimming methods can potentially be used to modify the resonators after fabrication. During this year, we continued to study a trimming method based on electrical field induced local oxidation of $\mathrm{Si}$ to $\mathrm{SiO}_{2}$ via a chemical reaction near an electrically-biased conducting atomic-force microscope tip [31], [37]. Scanning probe lithography has previously been used to modify the resonance frequency of a GaAs photonic crystal cavity, and is part of a larger class of methods developed for precision physical and chemical surface modification [38].

We continued to study and develop both the single-resonator and coupledresonator configurations for quantum light generation using spontaneous fourwave mixing [23]. The resonator-enhanced nonlinearity benefits the generation of entangled photon-pair and heralded single-photon generation using silicon photonics [1], [25], [39]. A single silicon microresonator can be monitored and controlled more easily [40] than a long CROW device and an optimized single silicon microresonator can also be useful for nonlinear wavelength conversion with low pump powers [41].

We require an external control "knob" to tune the waveguiding properties of the CROW structure. We decided to use electro-optic tunability for most of our studies. Using electro-optic control fields was practically convenient since it was easy to generate the switching waveforms in the laboratory [42]. If tuning speed is not a concern, the thermo-optic effect, which we used for tunable filters, is also another option as a control knob [43].

\section{Open-Access Reporting Initiative}

PRAISE: This open-access document is provided in support of our PRAISE

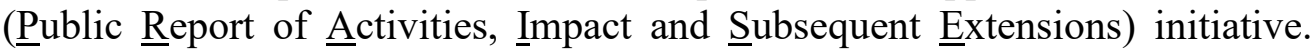
What is it? An open-access document shared with the public which describes the research outcomes of publicly-funded projects. For us, these projects are typically funded by the NSF (National Science Foundation).

\section{References}

[1] R. Kumar, M. Savanier, J. R. Ong, and S. Mookherjea, "Entanglement measurement of a coupled silicon microring photon pair source," Opt. Express, vol. 23, no. 15, p. 19318, Jul. 2015, doi: 10.1364/OE.23.019318.

[2] R. Kumar, J. R. Ong, J. Recchio, K. Srinivasan, and S. Mookherjea, "Spectrally multiplexed and tunable-wavelength photon pairs at $155 \mu \mathrm{m}$ 
from a silicon coupled-resonator optical waveguide," Opt. Lett., vol. 38, no. 16, p. 2969, Aug. 2013, doi: 10.1364/OL.38.002969.

[3] S. Mookherjea and A. Yariv, "Optical pulse propagation in the tightbinding approximation," Opt. Express, vol. 9, no. 2, p. 91, Jul. 2001, doi: 10.1364/OE.9.000091.

[4] S. Mookherjea, D. S. Cohen, and A. Yariv, "Nonlinear dispersion in a coupled-resonator optical waveguide," Opt. Lett., vol. 27, no. 11, p. 933, Jun. 2002, doi: 10.1364/OL.27.000933.

[5] S. Mookherjea and A. Oh, "Effect of disorder on slow light velocity in optical slow-wave structures," Opt. Lett., vol. 32, no. 3, p. 289, Feb. 2007, doi: 10.1364/OL.32.000289.

[6] S. Mookherjea and A. Yariv, "Pulse propagation in a coupled resonator optical waveguide to all orders of dispersion," Phys. Rev. E, vol. 65, no. 5, p. 056601, Apr. 2002, doi: 10.1103/PhysRevE.65.056601.

[7] M. L. Cooper et al., "Waveguide dispersion effects in silicon-on-insulator coupled-resonator optical waveguides," Opt. Lett., vol. 35, no. 18, p. 3030, Sep. 2010, doi: 10.1364/OL.35.003030.

[8] S. Mookherjea, "Dispersion characteristics of coupled-resonator optical waveguides," Opt. Lett., vol. 30, no. 18, p. 2406, Sep. 2005, doi: 10.1364/OL.30.002406.

[9] S. Mookherjea and A. Yariv, "Second-harmonic generation with pulses in a coupled-resonator optical waveguide," Phys. Rev. E, vol. 65, no. 2, p. 026607, Jan. 2002, doi: 10.1103/PhysRevE.65.026607.

[10] S. Mookherjea and A. Yariv, "Kerr-stabilized super-resonant modes in coupled-resonator optical waveguides," Phys. Rev. E, vol. 66, no. 4, p. 046610, Oct. 2002, doi: 10.1103/PhysRevE.66.046610.

[11] S. Mookherjea and A. Yariv, "Optical pulse propagation and holographic storage in a coupled-resonator optical waveguide," Phys. Rev. $E$, vol. 64, no. 6, p. 066602 , Nov. 2001, doi: 10.1103/PhysRevE.64.066602.

[12] S. Mookherjea, "Semiconductor coupled-resonator optical waveguide laser," Appl. Phys. Lett., vol. 84, no. 17, pp. 3265-3267, Apr. 2004, doi: 10.1063/1.1719278.

[13] J. E. Heebner, R. W. Boyd, and Q.-H. Park, "SCISSOR solitons and other novel propagation effects in microresonator-modified waveguides," J. Opt. Soc. Am. B, vol. 19, pp. 722-731, 2004.

[14] A. Ciattoni, B. Crosignani, S. Mookherjea, and A. Yariv, "Nonparaxial dark solitons in optical Kerr media," Opt. Lett., vol. 30, no. 5, p. 516, Mar. 2005, doi: 10.1364/OL.30.000516.

[15] B. Crosignani, A. Yariv, and S. Mookherjea, "Nonparaxial spatial solitons and propagation-invariant pattern solutions in optical Kerr media," Opt. Lett., vol. 29, no. 11, p. 1254, Jun. 2004, doi: 10.1364/OL.29.001254. 
[16] D. N. Christodoulides and N. K. Efremidis, "Discrete temporal solitons along a chain of nonlinear coupled microcavities embedded in photonic crystals," Opt. Lett., vol. 27, no. 8, pp. 568-570, 2002.

[17] R. Aguinaldo, Yiran Shen, and S. Mookherjea, "Large Dispersion of Silicon Directional Couplers Obtained via Wideband Microring Parametric Characterization," IEEE Photon. Technol. Lett., vol. 24, no. 14, pp. 1242-1244, Jul. 2012, doi: 10.1109/LPT.2012.2198639.

[18] J. R. Ong, R. Kumar, and S. Mookherjea, "Silicon microring-based wavelength converter with integrated pump and signal suppression," Opt. Lett., vol. 39, no. 15, p. 4439, Aug. 2014, doi: 10.1364/OL.39.004439.

[19] S. Mookherjea, "Using gain to tune the dispersion relation of coupledresonator optical waveguides," IEEE Photon. Technol. Lett., vol. 18, no. 5, pp. 715-717, Mar. 2006, doi: 10.1109/LPT.2006.871144.

[20] M. L. Cooper and S. Mookherjea, "Numerically-assisted coupledmode theory for silicon waveguide couplers and arrayed waveguides," Opt. Express, vol. 17, no. 3, p. 1583, Feb. 2009, doi: 10.1364/OE.17.001583.

[21] S. Mookherjea, "Spectral characteristics of coupled resonators," $J$. Opt. Soc. Am. B, vol. 23, no. 6, p. 1137, Jun. 2006, doi: 10.1364/JOSAB.23.001137.

[22] S. Mookherjea and M. A. Schneider, "The nonlinear microring adddrop filter," Opt. Express, vol. 16, no. 19, p. 15130, Sep. 2008, doi: 10.1364/OE.16.015130.

[23] J. R. Ong and S. Mookherjea, "Quantum light generation on a silicon chip using waveguides and resonators," Opt. Express, vol. 21, no. 4, p. 5171, Feb. 2013, doi: 10.1364/OE.21.005171.

[24] W. J. Kim, W. Kuang, and J. D. O'Brien, "Dispersion characteristics of photonic crystal coupled resonator optical waveguides," Opt. Express, vol. 11, pp. 3431-3437, 2003.

[25] M. Davanço et al., "Telecommunications-band heralded single photons from a silicon nanophotonic chip," Appl. Phys. Lett., vol. 100, no. 26, p. 261104, Jun. 2012, doi: 10.1063/1.4711253.

[26] T. F. Krauss, "Slow light in photonic crystal waveguides," J. Phys. D: Appl. Phys., vol. 40, no. 9, pp. 2666-2670, May 2007, doi: 10.1088/00223727/40/9/S07.

[27] T. Baba, "Slow light in photonic crystals," Nat. Photonics, vol. 2, no. 8, pp. 465-473, 2008.

[28] M. L. Cooper et al., "Statistics of light transport in 235-ring silicon coupled-resonator optical waveguides," Opt. Express, vol. 18, no. 25, p. 26505, Dec. 2010, doi: 10.1364/OE.18.026505.

[29] J. R. Ong et al., "Low-power continuous-wave four-wave mixing in silicon coupled-resonator optical waveguides," Opt. Lett., vol. 36, no. 15, pp. 2964-2966, 2011. 
[30] M. L. Cooper, G. Gupta, J. S. Park, M. A. Schneider, I. B. Divliansky, and S. Mookherjea, "Quantitative infrared imaging of silicon-on-insulator microring resonators," Opt. Lett., vol. 35, no. 5, p. 784, Mar. 2010, doi: 10.1364/OL.35.000784.

[31] Y. Shen, I. B. Divliansky, D. N. Basov, and S. Mookherjea, "Electricfield-driven nano-oxidation trimming of silicon microrings and interferometers," Opt. Lett., vol. 36, no. 14, p. 2668, Jul. 2011, doi: 10.1364/OL.36.002668.

[32] M. L. Cooper et al., "235-ring Coupled-Resonator Optical Waveguides," in Conference on Lasers and Electro-Optics 2010, San Jose, California, 2010, p. CTuHH3. doi: 10.1364/CLEO.2010.CTuHH3.

[33] S. Mookherjea and M. A. Schneider, "Avoiding bandwidth collapse in long chains of coupled optical microresonators," Opt. Lett., vol. 36, no. 23, p. 4557, Dec. 2011, doi: 10.1364/OL.36.004557.

[34] M. L. Cooper and S. Mookherjea, "Modeling of Multiband Transmission in Long Silicon Coupled-Resonator Optical Waveguides," IEEE Photon. Technol. Lett., vol. 23, no. 13, pp. 872-874, Jul. 2011, doi: 10.1109/LPT.2011.2141657.

[35] S. Mookherjea and H. R. Grant, "High dynamic range microscope infrared imaging of silicon nanophotonic devices," Opt. Lett., vol. 37, no. 22, p. 4705, Nov. 2012, doi: 10.1364/OL.37.004705.

[36] C. Ma, X. Wang, V. Anant, A. D. Beyer, M. D. Shaw, and S. Mookherjea, "Silicon photonic entangled photon-pair and heralded single photon generation with CAR $>12,000$ and $\mathrm{g}^{\wedge}(2)(0)<0006$," Opt.

Express, vol. 25, no. 26, p. 32995, Dec. 2017, doi: 10.1364/OE.25.032995.

[37] Y. Shen, I. B. Divliansky, D. N. Basov, and S. Mookherjea, "Perfect set-and-forget alignment of silicon photonic resonators and interferometers," in Optical Fiber Communication Conference/National Fiber Optic Engineers Conference 2011, Los Angeles, California, 2011, p. PDPC3. doi: 10.1364/OFC.2011.PDPC3.

[38] R. Garcia, R. V. Martinez, and J. Martinez, "Nano-chemistry and scanning probe nanolithographies," Chem. Soc. Rev., vol. 35, no. 1, pp. 29-38, 2006, doi: 10.1039/B501599P.

[39] R. Kumar, J. R. Ong, M. Savanier, and S. Mookherjea, "Controlling the spectrum of photons generated on a silicon nanophotonic chip," Nat Commun, vol. 5, no. 1, p. 5489, Dec. 2014, doi: 10.1038/ncomms6489.

[40] M. Savanier, R. Kumar, and S. Mookherjea, "Optimizing photon-pair generation electronically using a $p-i-n$ diode incorporated in a silicon microring resonator," Appl. Phys. Lett., vol. 107, no. 13, p. 131101, Sep. 2015, doi: 10.1063/1.4932047.

[41] J. R. Ong, R. Kumar, R. Aguinaldo, and S. Mookherjea, "Efficient CW Four-Wave Mixing in Silicon-on-Insulator Micro-Rings With Active 
Carrier Removal," IEEE Photon. Technol. Lett., vol. 25, no. 17, pp. 1699 1702, Sep. 2013, doi: 10.1109/LPT.2013.2272521.

[42] S. Mookherjea, J. R. Ong, X. Luo, and L. Guo-Qiang, "Electronic control of optical Anderson localization modes," Nature Nanotech, vol. 9, no. 5, pp. 365-371, May 2014, doi: 10.1038/nnano.2014.53.

[43] R. Aguinaldo et al., "Wideband silicon-photonic thermo-optic switch in a wavelength-division multiplexed ring network," Opt. Express, vol. 22, no. 7, p. 8205, Apr. 2014, doi: 10.1364/OE.22.008205. 